\title{
Admittance of Annular Slot Antennas Radiating Into a Plasma Layer
}

\author{
Janis Galejs
}

\author{
Contribution from Applied Research Laboratory, Sylvania Flectronic Systems, a Division of Sylvania Elec- \\ tric Products Inc., 40 Sylvan Road, Waltham 54, Mass.
}

(Received September 9, 1963)

\begin{abstract}
The slot admittance is calculated for a slot radiating into a waveguide of radius 5 to $10 \lambda$. The radius of the wide waveguide is selected so that the slot admittance is equal to the admittance for a semi-infinite half space in absence of plasma. This slot admittance is an approximation to the admittance of an inhomogeneous half space in presence of a plasma layer. The calculated slot susceptance is shown to be nearly constant for various thicknesses of the plasma layer, while the slot conductance and radiation conductances are decreased by increasing plasma thickness. This approximate calculation appears to be particularly good for thin layers of electrically contrasting plasma $\left(\epsilon_{p} / \epsilon_{0} \neq 1\right.$ and or $\left.\tan \delta_{p}>>1\right)$. The calculations indicate an admittance similar to that of earlier calculations for a rectangular slot radiating into a wide rectangular waveguide.
\end{abstract}

\section{Introduction}

The admittance of annular slots which are backed by coaxial lines or cavities and which radiate into free space have been discussed by Levine and Papas [1951]; Wait [1958]; Cohn and Flesher [1958], and Galejs and Thompson [1962]. The same formulation applies also to slots radiating into a half space filled with a homogeneous dielectric or plasma. There are difficulties in treating the inhomogeneous half space or dielectric and plasma layers of finite thicknesses, although such an analysis has been carried out by Galejs [1963a] for a rectangular slot covered by finite plasma layers. The calculations of the slot admittances are greatly simplified if the slot is assumed to radiate into a wide waveguide instead of an inhomogeneous half space [Galejs, 1963b]. A waveguide of a finite diameter is characterized by discrete modes which can be readily treated in machine calculations.

Radiation from a coaxial line into a waveguide of infinite diameter has been discussed by Cohn and Flesher [1958]. Their results agree with an analytical formulation not employing such artificial boundaries [Levine and Papas, 1951]. In the present note a waveguide of finite diameter will be used and it will be shown that the admittance of this waveguide approximates the admittance for an inhomogeneous half space. This was also the case for rectangular slots provided the guide diameter exceeded 5 to $10 \lambda$ [Galejs, 1963b]. A similar behavior can be also expected for annular slots radiating into a circular guide because the fields in the vicinity of the slot should not be affected by the presence of the waveguide walls if the distance between the walls is large enough. Hence, this geometry will yield realistic susceptances and also realistic estimates of losses in the plasma layer. However, the radiation fields of the slot will be modified by the waveguide walls. The radion patterns of the slot cannot be determined in this model and the effects of guide diameter on the radiation conductance must be also examined in detail.

The analytical formulation of the admittance for a cavity backed slot can be considered as straightforward, and only the principal steps of the development are summarized in section 2. Convergence considerations and the numerical results will be discussed in section 3 .

\section{Slot Admittance}

The waveguide and slot geometry is shown in figure 1. The same basic geometry has been considered by Galejs and Thompson [1962], and the configuration of figure 1 may be treated by a suitable modification of this work. For an exp $(j \omega t)$ time variation of the $\phi$ symmetrical fields the magnetic field components $H_{\phi}(\rho, z)$ are given by

$$
\begin{aligned}
& H_{\phi i}=\sum_{m=1}^{\infty} A_{m} R_{m}(\rho)\left(e^{-\gamma_{m} z}+B_{m} e^{\gamma} m^{z}\right) \\
& H_{\phi j}=\sum_{n=1}^{\infty} A_{n j} R_{n}(\rho)\left(e^{-\gamma_{n j} z}+B_{n j} e^{\gamma_{n j^{z}}}\right)
\end{aligned}
$$

where $j=0,1$ or 2 and where $B_{n j}=0$. The orthonormal radial functions $R_{p}(\rho)$ are defined as

$$
R_{p}(\rho)=N_{p} J_{1}\left(\lambda_{p} \rho\right)
$$

where $p=m$ or $n$ and

$$
J_{0}\left(\lambda_{m} b\right)=J_{0}\left(\lambda_{n} c\right)=0
$$




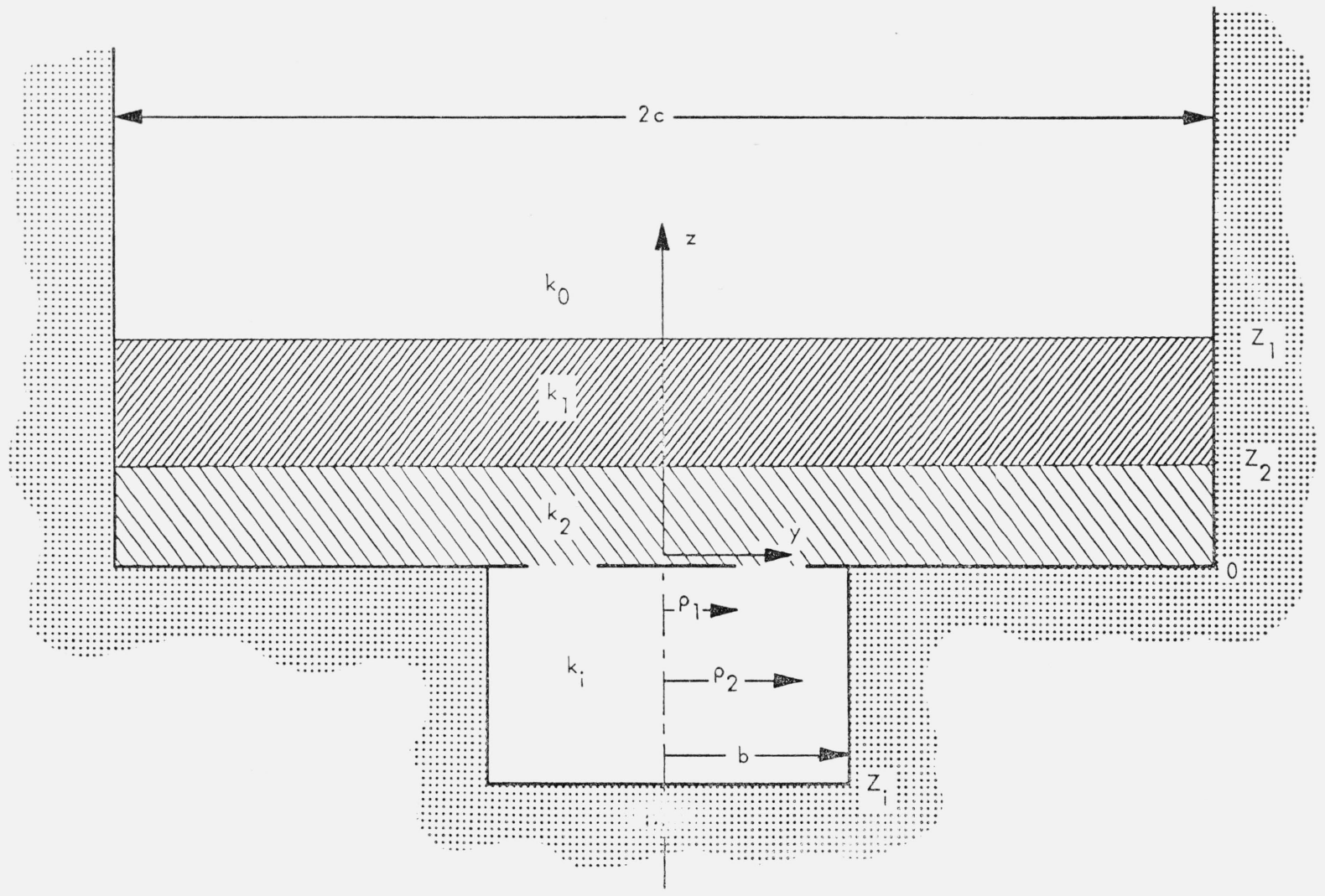

Figure 1. Slot geometry.

$$
\begin{aligned}
& N_{m}^{-1}=\frac{b}{\sqrt{2}} J_{1}\left(\lambda_{m} b\right) . \\
& N_{n}^{-1}=\frac{c}{\sqrt{2}} J_{1}\left(\lambda_{n} c\right)
\end{aligned}
$$

The propagation constants $\gamma_{m}$ and $\gamma_{n j}$ are defined in the first quadrant of the complex plane by

where

$$
\begin{aligned}
\gamma_{m} & =\sqrt{\lambda_{m}^{2}-k_{i}^{2}} \\
\gamma_{n j} & =\sqrt{\lambda_{n}^{2}-k_{j}^{2}}
\end{aligned}
$$

$$
k_{j}^{2}=\omega^{2} \mu_{0} \epsilon_{j}-j \omega \mu_{0} \sigma_{j}
$$

with a corresponding expression for $k_{i}^{2}$. The radial electric field component $E_{\rho}$ is computed from (1) or (2) as

$$
\left(\sigma_{j}+j \omega \epsilon_{j}\right) E_{\rho j}(\rho, z)=-\frac{\partial}{\partial t} H_{\phi j}(\rho, z)
$$

where $j=0,1,2$, or $i$. The amplitudes $A_{q}(q=m$ or $n 2)$ are related to the excitation field of the cavity $E_{\rho}(\rho, 0)=\mathscr{E}(\rho)$. Multiplying $E_{\rho}(\rho, 0)$ as computed from (10) with $R_{p}(\rho) \rho d \rho$ and integrating over the $z=0$ plane gives

$$
A_{q}=\frac{\left(\sigma_{j}+j \omega \epsilon_{j}\right)}{\left(1-B_{q}\right) \gamma_{q}} \int_{\rho_{1}}^{{ }^{\prime} z} \mathscr{E}(\rho) R_{p}(\rho) \rho d \rho
$$

with $j=i$ or 2 . The tangential magnetic fields $H_{\phi i}(\rho, 0)$ and $H_{\phi 2}(\rho, 0)$ differ by the linear source current density $J_{s}(\rho)$ across the slot, where $J_{s}(\rho)$ is assumed to be of the form

$$
J_{s}(\rho)=\frac{I}{2 \pi \rho} .
$$

After substituting (11) in (1) and (2) one obtains arı integral equation

$$
H_{\phi i}(\rho, 0)=H_{\phi 2}(\rho, 0)+J_{s}(\rho)
$$

in the unknown field distribution $\mathscr{E}(\rho)$. A variational principle for determining $\mathscr{E}(\rho)$ is constructed by multiplying (13) with $\rho \mathscr{E}(\rho)$ and then integrating from $\rho=\rho_{1}$ to $\rho_{2}$. After defining the voltage across the slot $V$ as

$$
V=-\int_{\rho_{1}}^{\rho_{2}} \mathscr{E}(\rho) d \rho
$$


it follows that

$$
\begin{aligned}
\frac{I V}{2 \pi} & =\sum_{n=1}^{\infty} \frac{\sigma_{2}+j \omega \epsilon_{2}}{\gamma_{n 2}} \frac{1+B_{n 2}}{1-B_{n 2}}\left[\int_{\rho_{1}}^{\rho_{2}} \mathscr{E}(\rho) R_{n}(\rho) \rho d \rho\right]^{2} \\
& -\sum_{m=1}^{\infty} \frac{\sigma_{i}+j \omega \epsilon_{i}}{\gamma_{m}} \frac{1+B_{m}}{1-B_{m}}\left[\int_{\rho_{1}}^{\rho_{2}} \mathscr{E}(\rho) R_{m}(\rho) \rho d \rho\right]^{2} .
\end{aligned}
$$

The integrations can be carried out for a general power series representation of $\mathscr{E}(\rho)$ and it is formally possible to determine all the coefficients in this power series. [Levine and Papas, 1951; Galejs and Thompson, 1962.] However, the subsequent numerical work appears to be tractable only for the simplest form of the trial function

$$
\mathscr{E}(\rho)=\frac{a_{0}}{\rho}
$$

The integrations are straightforward for this form of $\mathscr{E}(\rho)$ and the slot admittance $Y=I / V$ follows as:

$$
\begin{aligned}
Y= & \frac{4 \pi}{\left(\log \frac{\rho_{2}}{\rho_{1}}\right)^{2}}\left\{\sum_{n=1}^{\infty} \frac{\sigma_{2}+j \omega \epsilon_{2}}{\gamma_{n 2}} \frac{1+B_{n 2}}{1-B_{n 2}}\right. \\
& {\left[\frac{J_{0}\left(\lambda_{n} \rho_{1}\right)-J_{0}\left(\lambda_{n} \rho_{2}\right)}{\lambda_{n} c J_{1}\left(\lambda_{n} c\right)}\right]^{2}-\sum_{m=1}^{\infty} \frac{\sigma_{i}+j \omega \epsilon_{i}}{\gamma_{m}} \frac{1+B_{m}}{1-B_{m}} } \\
& {\left.\left[\frac{J_{0}\left(\lambda_{m} \rho_{1}\right)-J_{0}\left(\lambda_{m} \rho_{2}\right)}{\lambda_{m} b J_{1}\left(\lambda_{m} b\right)}\right]^{2}\right\} . }
\end{aligned}
$$

The reflection coefficient $B_{m}$ is determined from the requirement that $E_{\rho}\left(\rho, z_{0}\right)=0$. This gives

$$
B_{m}=e^{-2 \gamma_{m} z_{i}} .
$$

The reflection coefficient $B_{n j}$ is determined after matching the $E_{\rho}$ and $H_{\phi}$ components across the dielectric interfaces at $z=z_{1}$ and $z_{2}$. A computation results in

$$
B_{n j}=e^{-2 \gamma_{n j} z_{j}} \quad \frac{S_{n j}-D_{n j}}{S_{n j}+D_{n j}}
$$

where

$$
\begin{gathered}
S_{n j}=\left(\frac{k_{j-1}}{k_{j}}\right)^{2}\left[e^{-2 \gamma_{n(j-1)} z_{j}}+B_{n(j-1)}\right] \\
D_{n j}=\frac{\gamma_{n(j-1)}}{\gamma_{n j}}\left[e^{-2 \gamma_{n(j-1)} z_{j}-} B_{n(j-1)}\right]
\end{gathered}
$$

and where $j=1$ or 2 . The computation starts by determining $B_{n 1}$ with $B_{n 0}=0$ (there are only outgoing waves for $z>z_{1}$ ). This value of $B_{n 1}$ is then used for computing $B_{n 2}$, which completes the determination of the slot admittance.

As long as $\sigma_{i}, \sigma_{1}$, or $\sigma_{2} \neq 0$, the real part of $Y$ will differ from the radiation conductance $G_{r}$. However, $G_{r}$ may be determined after computing the power flow for $z>z_{1}$. The radiated power is given by

$$
\begin{aligned}
P_{r} & =G_{r} V^{2} \\
& =\operatorname{Re} \iint E_{\rho 0} \times H_{\phi 0}^{*} \rho d \rho d \phi \\
& =2 \pi \operatorname{Re} \sum_{n} \frac{\gamma_{n 0}}{j \omega \epsilon_{0}} \mid e^{-\left.\gamma_{n 0^{z}} A_{n 0}\right|^{2} .}
\end{aligned}
$$

The only values of $n$ which contribute to $P_{r}$ are those with imaginary $\gamma_{n 0}$. Hence $\mid e^{-\gamma_{n 0} z^{2}}=1$ and

$$
G_{r}=2 \pi \operatorname{Re} \sum_{n} \frac{\gamma_{n 0}}{j \omega \epsilon_{0}}\left|\frac{A_{n 0}}{V}\right|^{2},
$$

$A_{n 0}$ is related to $A_{n 2}$ by matching either $H_{\phi}$ or $E_{\rho}$ across the dielectric boundaries at $z=z_{1}$ and $z_{2}$. It follows that

$$
\frac{A_{n 0}}{A_{n 2}}=\frac{e^{-\gamma} 1^{z_{1}}+B_{n 1} e^{\gamma} n 1^{z_{1}}}{e^{-\gamma_{n 0} z_{1}}} \frac{e^{-\gamma_{n 2^{z_{2}}}}+B_{n 2} e^{\gamma_{n 2^{z_{2}}}}}{e^{-\gamma_{n 1} z_{2}}+B_{n 1} e^{\gamma} 1^{1_{2}}}
$$

where $B_{n 1}$ and $B_{n 2}$ are obtained from (19) to (21). The amplitude $A_{n 2}$ is related to $V$ from (11), (14), and (16) as

$$
\frac{A_{n 2}}{V}=-\frac{\sqrt{2}\left(\sigma_{2}+j \omega \epsilon_{2}\right)\left[J_{0}\left(\lambda_{n} \rho_{1}\right)-J_{0}\left(\lambda_{n} \rho_{2}\right)\right]}{\left(\log \frac{\rho_{2}}{\rho_{1}}\right)\left(1-B_{n 2}\right) \gamma_{n 2} \lambda_{n} c J_{1}\left(\lambda_{n} c\right)} .
$$

The substitution of (24) and (25) in (23) completes the specification of $G_{r}$.

\section{Discussion}

The slot admittance is examined first for radiation into a homogeneous large guide of increasing radius $c$. The plot of the slot conductance in figure 2 is discontinuous with discontinuities occurring at those points where an additional mode starts propagating in the large guide. The slot susceptance, not shown in figure 2, exhibits discontinuities at the same points and large susceptances occur below the cut-off radius of the guide when $\gamma_{\text {no }}$ is real and approaches zero. (This behavior of the susceptance does not follow from the elementary considerations of sec. 1.) The slot admittance for the radiation into a homogeneous half space is shown dashed in figure 2. The conductance curves for the waveguide cross periodically the half-space conductance curve. The changes of the waveguide admittance tend to become less for increasing guide radius $c$, and a randomly selected guide radius will give a fair approximation to the correct admittance if $c$ is of the order of 15 to $30 \lambda$. However, the relatively slow convergence of the sum representing the slot susceptance restricts the choice of $c$ to smaller values. For smaller guides 


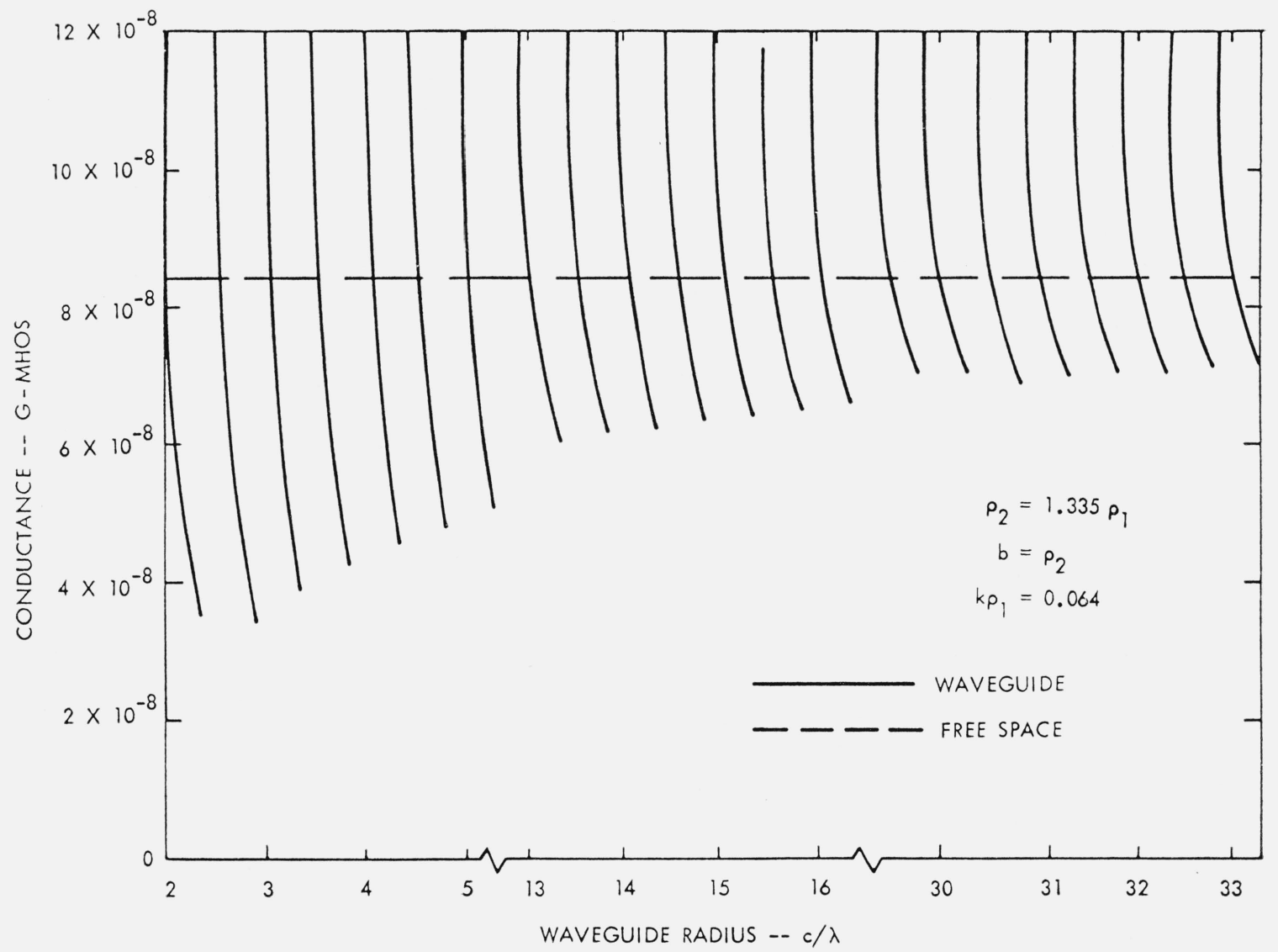

FiguRE 2. Conductance of the annular slot.

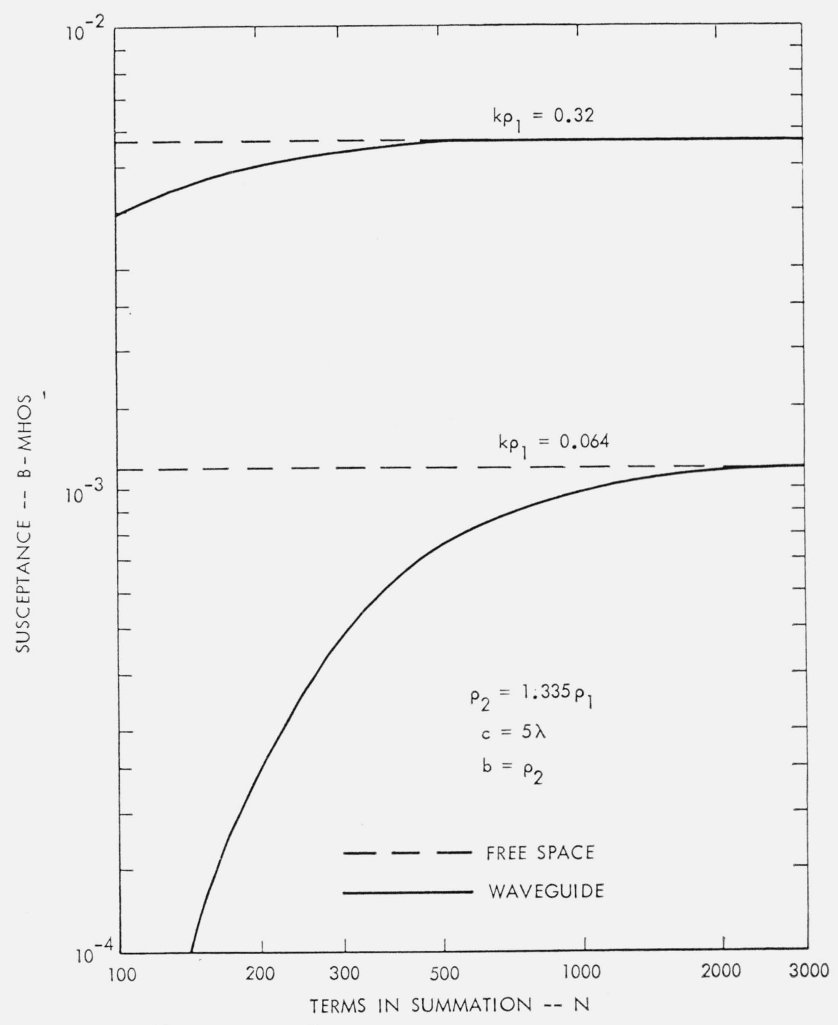

Figure 3. Susceptance of the annular slot.

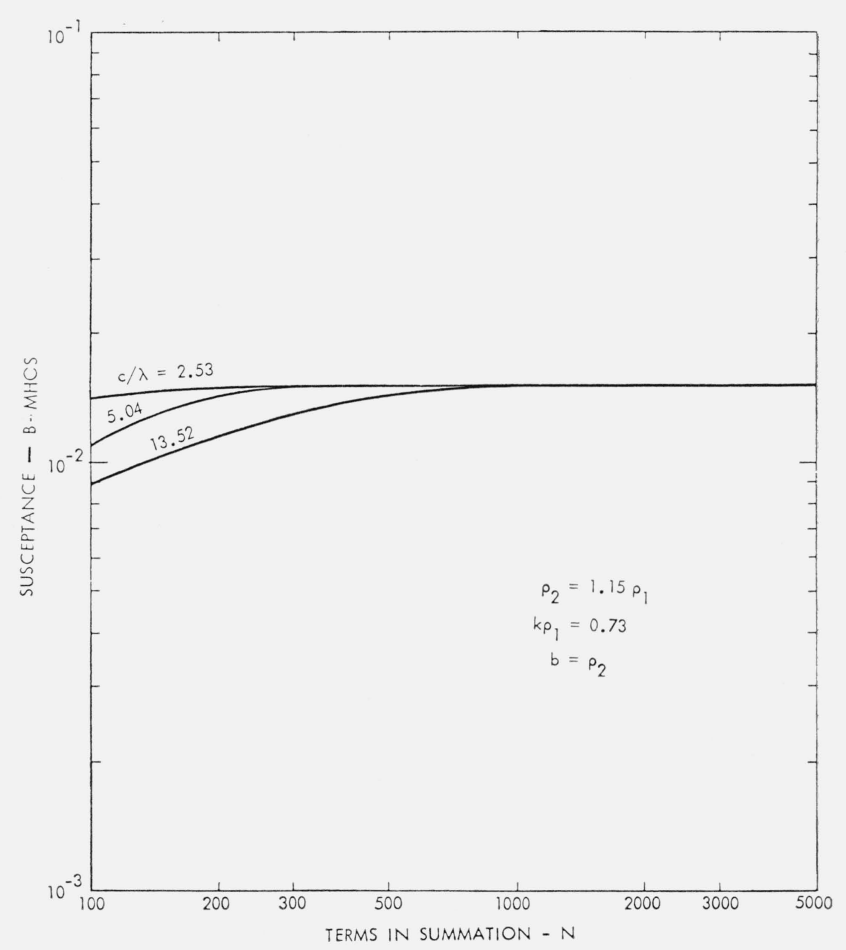

Figure 4. Susceptance of the annular slot. 
the radius may be always selected such that the waveguide admittance is equal to the slot admittance for a homogeneous half space. The susceptance calculations are illustrated in figure 3 for two values of slot radius $\rho_{1}$. For small guide radii the series converges slowly, and a larger number of terms will be required in the summation. Similarly, a larger number of terms is required for guides of large radii $c$, as is evident from figure 4 .

Admittance calculations for plasma layers of various thicknesses $h$ are illustrated in figures 5 and 6 . The slot conductance $G$ and the radiation conductance $G_{r}$ decrease with increasing layer thicknesses $h$, while the slot susceptance is almost constant for $h / \lambda>0.1$. For plasma layer of $h / \lambda>0.3$, the conductance depends on the guide radius $c$. The computed conductance is more accurate for the larger

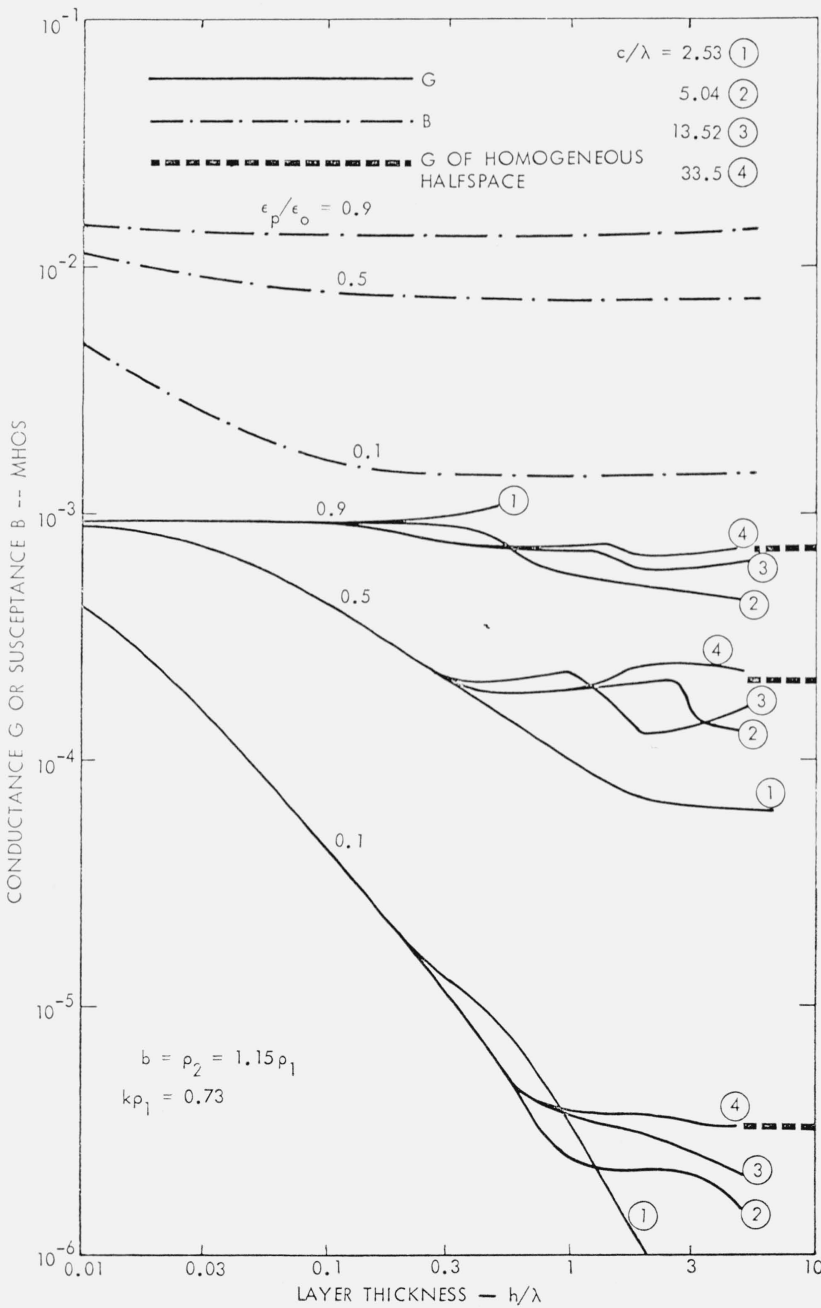

Figure 5. Slot admittance for loss less plasma layers.

${ }^{1}$ For $k \rho_{1}<<1$ and $\rho_{2} / \rho_{1} \approx 1$ and for a lossless dielectric half space

$$
G_{0}=\sqrt{\frac{\epsilon}{\epsilon_{0}}} \frac{(k \rho)^{4}}{360}\left[1-\frac{(k \rho)^{2}}{5}+\frac{(k \rho)^{4}}{56}+\cdots\right]
$$

where $\rho=\left(\rho_{1}+\rho_{2}\right) / 2$. For a lossy dielectric half space $G_{1}=G_{0}+B \tan \delta$. $c$ values when it approaches $G$ of the homogeneous half space ${ }^{1}$ for $h / \lambda>1$. A similar effect of increasing waveguide size was also observed for rectangular plasma covered slots [Galejs, 1963b]. For slots covered by thin plasma layers the admittance is principally determined by the nearby air-to-plasma interface, and is hardly affected by the relatively distant guide walls. The guide walls have an effect for large plasma thicknesses, particularly in cases where $\epsilon_{p} / \epsilon_{0}$ is near unity, or where the electrical discontinuity at the air-to-plasma interface is small.

Only the admittance reflected into the slot plane by the outside space $(z>0)$ has been indicated in figures 5 and 6 . The admittance reflected by the cavity or $Y_{i}$ has been discussed earlier by Galejs and Thompson [1962], and is not included in the above admittance figures.

Slot admittance calculations will be also made for the parameters of a typical reentry plasma. An example of the electron density $N_{i}$ and collision frequency $\nu$ profiles near a hypersonic reentry vehicle is given in figure 19 of Rotman and Meltz [1961]. These data have been replotted in figure 7 along with

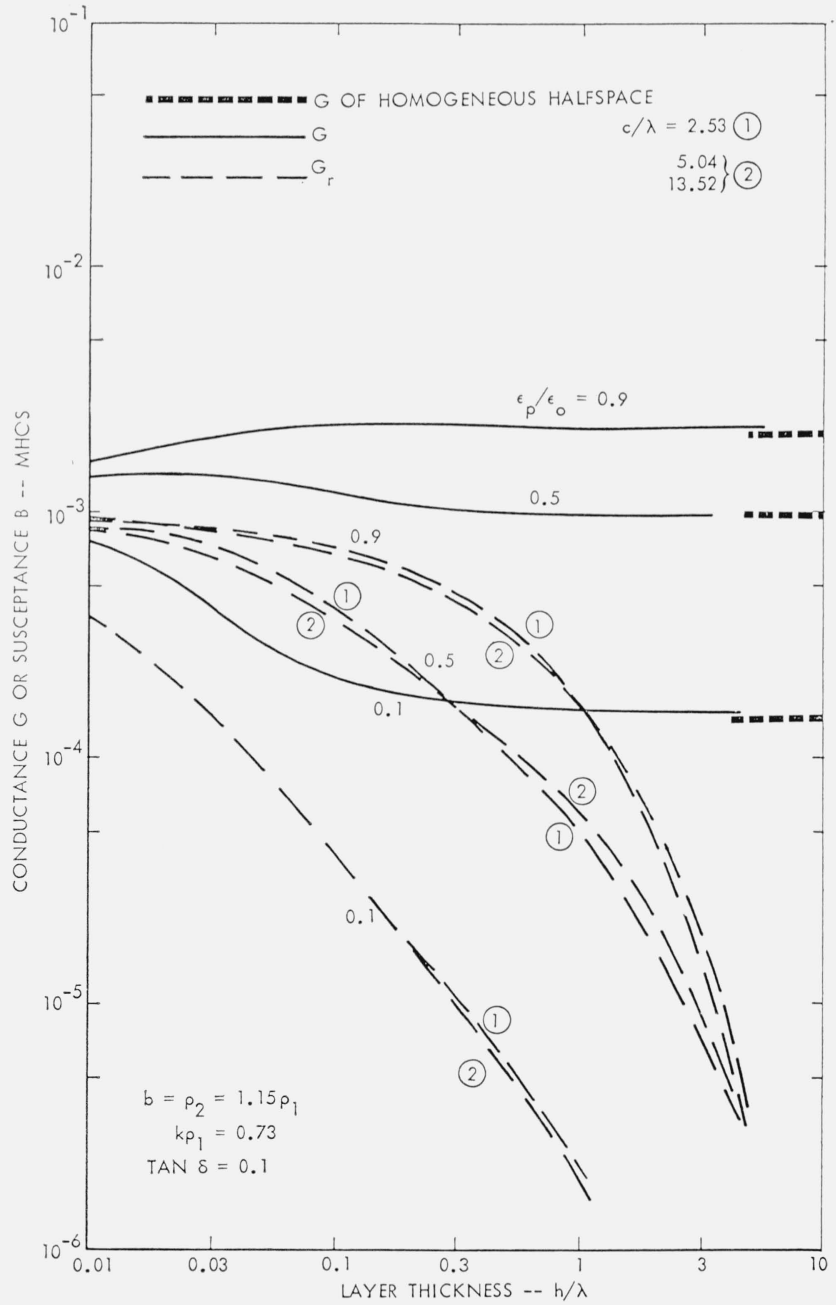

Figure 6. Slot admittance for lossy plasma layers. 


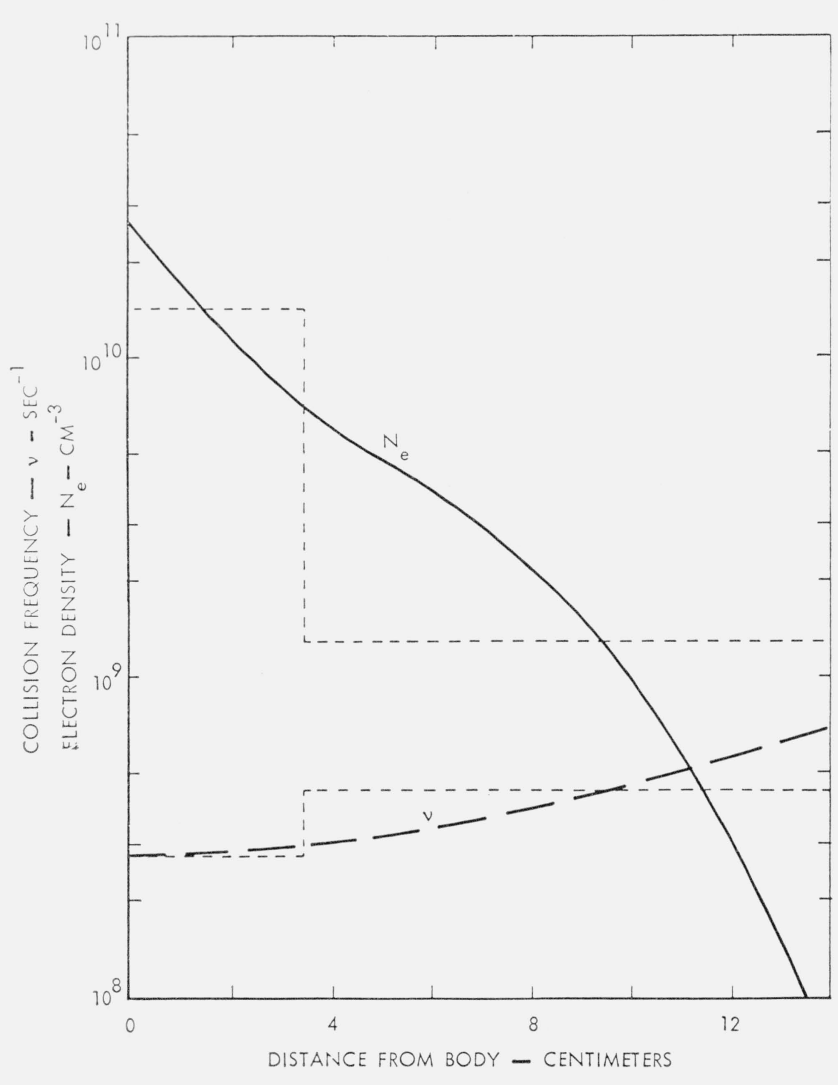

Figure 7. Profile of electron density and collision frequency and the two layer approximation.

Velocity of $17.5 \mathrm{~K}$ fps and Altitude of $200 \mathrm{~K} \mathrm{ft}$.

the two-layer approximation that is suited for the present calculation. The calculated slot admittance is shown in figure 8 for various operating frequencies while the slot and the waveguide dimension remain constant in relation to the operating wavelength. For frequencies above the plasma frequency $\left(f>f_{p}\right)$ the radiation conductance is nearly the same as the total slot conductance. For frequencies below the plasma frequency the slot susceptance $B$ is inductive and there are high losses and a low radiation conductance. The radiation conductance exhibits dips near the plasma frequencies of the two layers, which are at $f_{p}=3.22 \times 10^{8}$ and $1.06 \times 10^{9} \mathrm{c} / \mathrm{s}$. Obviously, this example does not cover all the plasma and slot parameters of interest in reentry problems. However, the above calculations are straightforward and further examples will not be considered at present.

The present model is also suited for determining the slot admittance in presence of lossy dielectric layers. In an example the layer near the slot is assumed to be of low losses $\left(\tan \delta_{2}=0.01\right)$, while the outer layer is highly lossy $\left(1 \leq \tan \delta_{1} \leq 100\right)$.

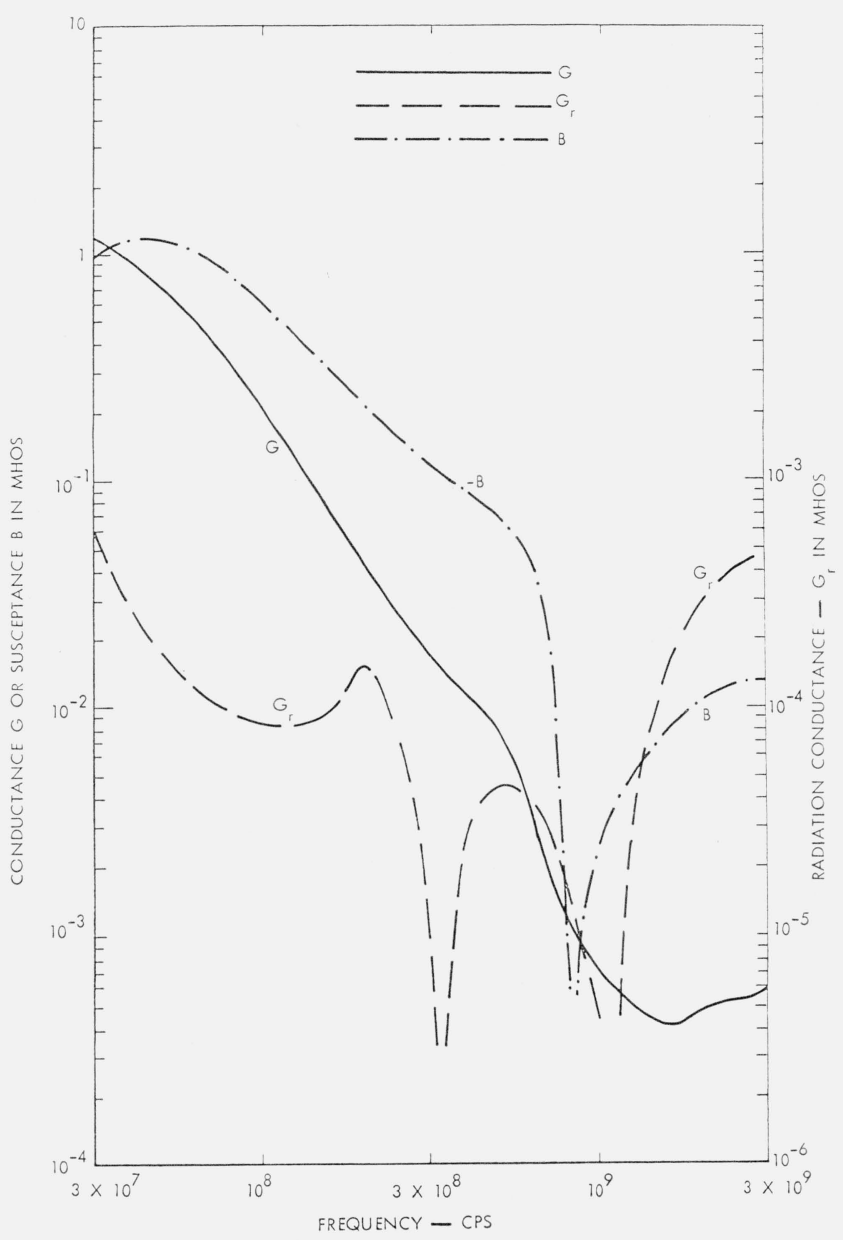

FIGURE 8. Slot admittance for a two layer approximation of a reentry plasma profile.

The calculated slot admittance is shown in figures 9 to 11 , for various distances $z_{2}$ between the slot and the lossy layer. For a given thickness $\left(z_{1}-z_{2}\right)$ of the lossy laver the losses and $G$ are highest for $z_{2}$ small, but the radiation conductance $G_{r}$ is essentially constant over a range of $z_{2}$ and $\left(z_{1}-z_{2}\right)$ values. For very thin lossy layers the capacitive susceptance $B$ approaches the free space value $B$ of figure 4 for $z_{2}$ small. For thick highly lossy layers $(\tan \delta=100)$ $B$ is highest for $z_{2}$ small.

The above waveguide model provided convenient means for analyzing slot and plasma or dielectric configurations that would appear untractable in more exact geometries. The susceptance and loss computations can be iustified either by reasoning that distant guide walls do not perturb near fields of the slot or by comparing the admittance in the limit of very thick or thin plasma layers with known results for a homogeneous half space or free space. The dimensions of the large guide may be also selected in such a way that the calculated radiation conductance agrees in the same limiting cases with known 


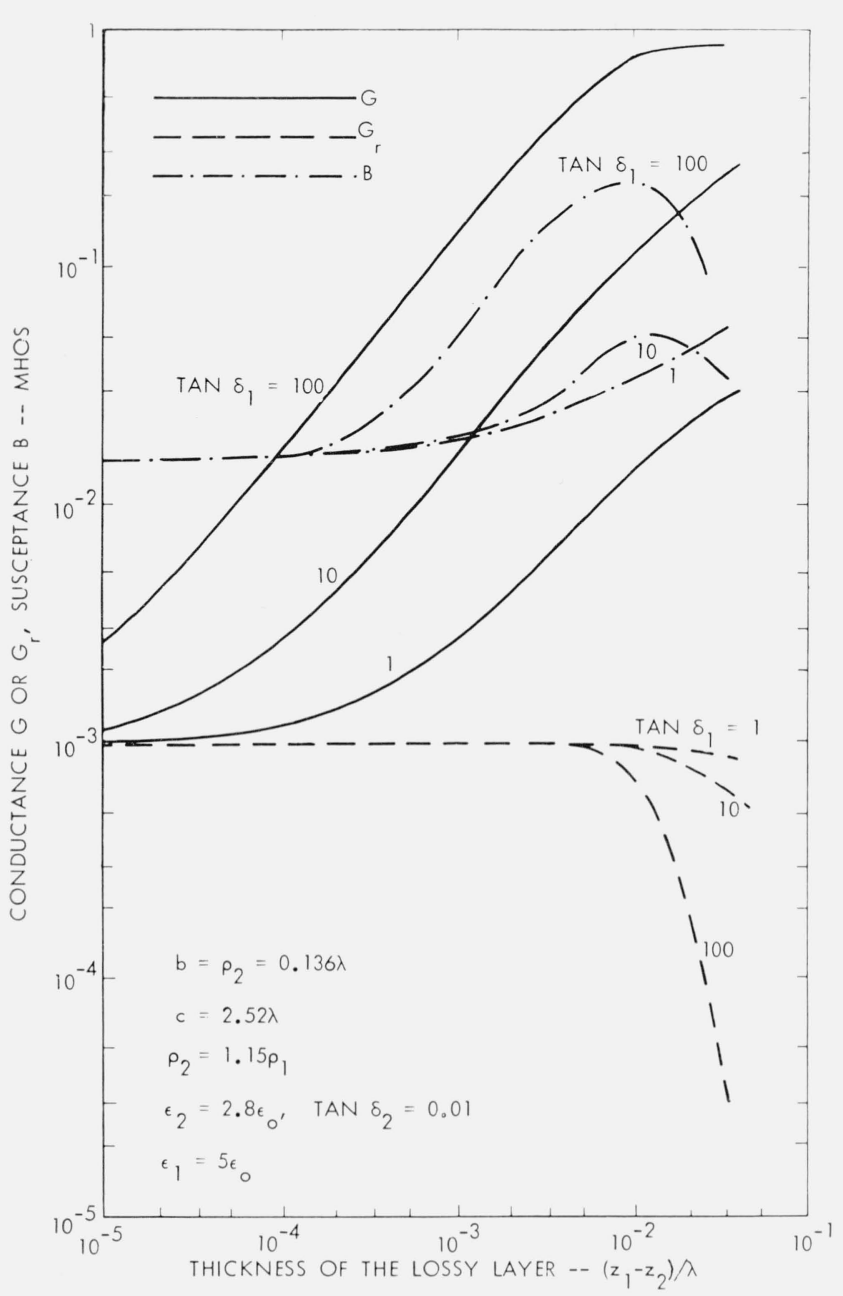

Figure 9. Admittance of a slot covered by lossy dielectric at a distance $\mathrm{Z}_{2}=3 \times 10^{-4} \lambda$.

results. There are no other solutions for the intermediate range of layer thicknesses for the annular slot. However, for rectangular slots there are comparable solutions for waveguides and for inhomogeneous half space [Galejs, 1963a, b].

Appreciation is expressed to D. A. Breault of Sylvania's Applied Research Laboratory for computer programming. This research was sponsored by the Air Force Cambridge Research Laboratories, Office of Aerospace Research, under Contract No. AF19(628)-2410.

\section{References}

Cohn, G. I., and G. T. Flesher (1958), Theoretical radiation pattern and impedance of a flush-mounted coaxial aperture, Proc. Nat. Electron. Conf. 14, 150-168.

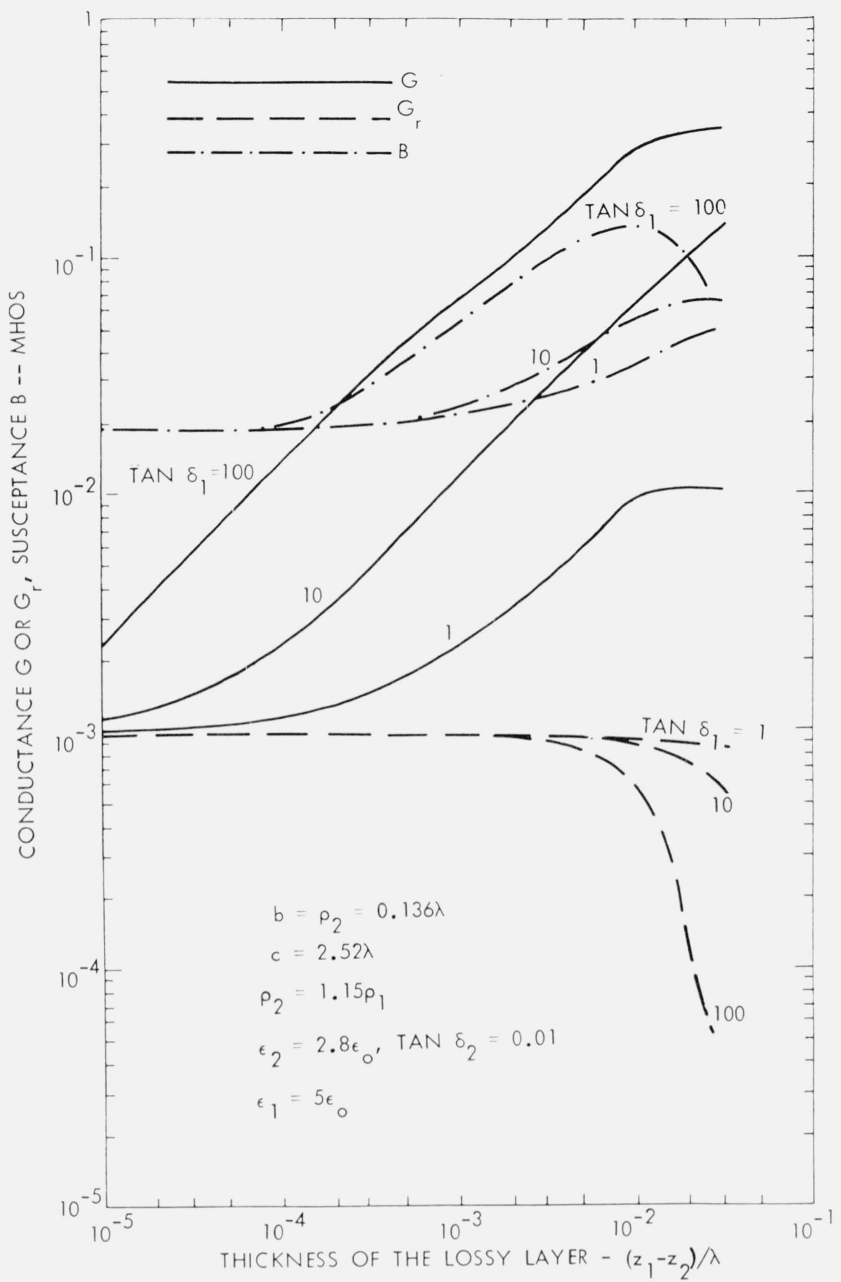

Figure 10. Admittance of a slot covered by lossy dielectric at a distance $\mathrm{Z}_{2}=3 \times 10^{-3} \lambda$.

Galejs, J., and T. W. Thompson (Nov. 1962), Admittance of a cavity-backed annular slot antenna, IRE Trans. Antennas Propagation AP-10, No. 6, 671-678.

Galejs, J. (June 1963a), Slot antenna impedance for plasma layers, Research Report No. 346, Applied Research Laboratory, Sylvania Electronic Systems.

Galejs, J. (June 6, 1963b), Admittance of a waveguide radiating into stratified plasma, Research Report No. 347, Applied Research Laboratory, Sylvania Electronic Systems.

Levine, H., and C. H. Papas (Jan. 1951), Theory of circular diffraction antenna, J. Appl. Phys. 22, 29-43.

Rotman, W., and G. Meltz (March 1961), Experimental investigation of the electromagnetic effects of re-entry, Report AFCRL 87, U.S. Air Force Cambridge Research Laboratories, Bedford, Mass.

Wait, J. R. (Jan. 1958), A low-frequency annular-slot antenna, J. Res. NBS 60, 59-64 RP 2822. 


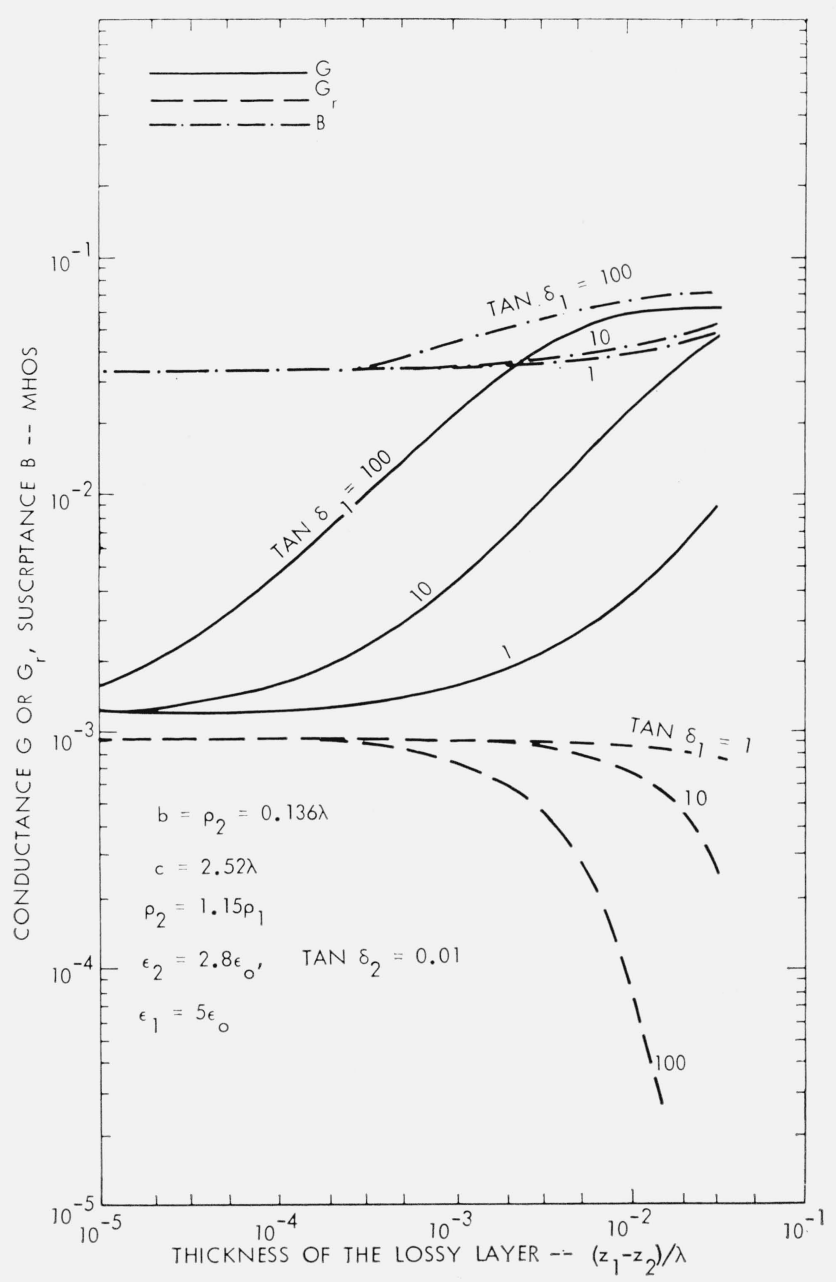

Figure 11. Admittance of a slot covered by lossy dielectric at a distance $\mathrm{Z}_{2}=3 \times 10^{-2} \lambda$.

(Paper 68D3-348) 